\title{
A Replicator Dynamics Method for the Unit Commitment Problem
}

\author{
Sohail Khan, Hadrien Bosetti and Peter Palensky \\ Austrian Institute of Technology, Energy Department, Vienna, Austria \\ sohail.khan.fl@ait.ac.at, \{givenname.surname\}@ait.ac.at
}

\author{
Wolfgang Gawlik \\ TU Wien - Austria \\ gawlik@ea.tuwien.ac.at
}

\begin{abstract}
A short-term Unit Commitment problem in power systems requires methods that are simple, stable and reconfigurable. A Replicator Dynamics based algorithm can be a good candidate in this regard. In this paper, this method is extended to model the unit specific constraints on the fitness function directly and precisely such that the method retains the optimality of the solution. Furthermore, the results are validated by comparison with Newtons Approach for optimal dispatch. The simulation results verify the methodology and implicate the possibility of extending it to complex and coupled constraints.
\end{abstract}

\section{INTRODUCTION}

Unit Commitment (UC) models the power pool auctions in a market scenario where for a given electricity demand each generation unit proposes a bid [1]. This bid consists of a cost function subject to the unit operational constraints. The selection of a bidding strategy can be dependent on the strategies of other units (i.e. players) and environmental variables. This strategic interaction can be modeled using Replicator Dynamics (RD) from the evolutionary game theory. RD models the dynamics of a population playing a specific strategy such that a successful strategy attracts more elements of population [2]. RD is an attractive choice for such problems as its benefits include reduced complexity, support for parallel computation and re-configurability.

A detailed review of various methods for UC problem can be found in [1]. The control decisions for short term horizon are influenced by increasing number and complexity of variables, such as partially deregulated market structure, transmission lines constraints to name a few. Particularly, the time dependent cost, uncertainty in forecasts and coupled constraint structure makes the problem hard to solve. Various methods are developed for such cases like mixed-integer programming [3] and fuzzy based methods [4]. Few hybrid techniques that combine heuristic techniques with dynamic programming are introduced in [1]. Among others, Non-smooth fuel cost functions are discussed in [5] and cooperative method combining Genetic Algorithm with Lagrangian relaxation is discussed in [6]. Among them, the non-deterministic techniques are computationally intensive while the deterministic techniques does not guarantee global optimality [7]. Thus its worthwhile to work on algorithms that are fast and leads to globally optimum solution.

In a deregulated market structure the players are not bound to fulfill the demand as conventionally. Rather, the bid is structured based on the demand and price is determined dynamically. So the objective of each player is to maximize the profit which may not be equivalent to cost minimization.
Therefore for system operator, it's important to model the strategic interaction in broader perspective.

$\mathrm{RD}$ based economic dispatch of distributed generators is discussed in [8], and it is extended to UC problem in this work. Specifically, the methodology of mapping the unit specific constraints on fitness function is presented. The result of optimal resource allocation using $\mathrm{RD}$ is validated by comparison with Newtons Approach (NA) for optimal dispatch. The result increases the confidence of extending the work to multi-objective scenarios. In last the fitness function dynamics are closely observed to explore the rate of convergence and dependency on initial conditions. Based on these results, the stability of the equilibrium is also discussed.

The article is organized as follows. The basic concept of $\mathrm{RD}$ and analogy with NA is introduced in Sec. II. It is followed by the problem formulation along with a brief discussion on the stability of the desired equilibrium. In Sec. III, results for our test case are presented. The paper is concluded in Sec. IV with a short summary.

\section{PRoblem Statement}

\section{A. About Replicator Dynamics and Newtons Approach}

$\mathrm{RD}$ equation models the dynamic resource allocation assuming that growth rate of strategy's frequency corresponds to the strategy's payoff, or more precisely, to the difference between its payoff $(A x)_{i}$ and the average payoff $(x . A x)$ in the population [9]

$$
\dot{x}_{i}=x_{i}\left[(A x)_{i}-(x . A x)\right],
$$

where $x_{i}$ is the population state of strategy $i$ and $A$ is the payoff matrix. This equation describes a selection process for strategies such that the most successful strategies spread in the population. To perform analogy with the UC problem we assume that there are $N$ controllable units that are modeled as $N$ habitats ( strategies) with each one having a fitness function. The total power demand at an instance is assumed fixed and it is regarded as a population that is searching for a habitat. The dynamics of power assigned, as population in a habitat, can be described analogous to Eq. (1) as follows,

$$
\dot{p}_{i}=p_{i}\left(f_{i}\left(p_{i}\right)-\bar{f}\right)
$$

where $f_{i}\left(p_{i}\right)$ and $\bar{f}$ represent the $i^{\text {th }}$ unit fitness value and the average fitness value, respectively. $f_{i}\left(p_{i}\right)$ is obtained from 
quadratic cost function of the unit as discussed in [8]. The discrete time version is given as follows,

$$
\begin{aligned}
p_{i}[m+1] & =p_{i}[m] \frac{1 / T_{s}+f_{i}[m]}{1 / T_{s}+\bar{f}[m]}, \\
f_{i}[m+1] & =\frac{1}{c_{i}}\left(1-\frac{p_{i}[m+1]}{p_{i}^{\text {nom }}}\right), \\
\bar{f}[m] & =\frac{1}{P_{d}^{\prime}} \sum_{j=1}^{N} p_{j}[m] f_{j}[m],
\end{aligned}
$$

where $m=1,2,3, \ldots$ is the iteration count for each time instance $[k], c_{i}$ is the cost factor specific to each unit and $N$ is the number of generator units. Eqs. (3) and (4) are computed at each unit local controller while Eq. (5) is calculated at the central node. The process continues until the individual fitness function values converge to the average fitness value:

$$
f_{i}\left(p_{i}\right)=\bar{f}
$$

The resultant solution is optimal, for details see [8] and [2]. The structure of RD has similar structure in comparison with NA for optimal power flow [10]. In this method the constraints are augmented to the cost function using Lagrange multipliers as,

$$
\mathcal{L}=C_{\text {total }}+\lambda\left(P_{d}^{\prime}-\sum_{i=1}^{N} p_{i}\right)
$$

where $C_{\text {total }}$ is the sum of cost function of all the units and $P_{d}^{\prime}$ is the total demand. The function is minimized for the following condition

$$
\frac{\partial C_{i}}{\partial P_{i}}=\lambda
$$

which is analogous to Eq. (6) stating that the generation dispatch is optimal if each unit operates at same incremental cost [11].

In Newtons method the value of $\lambda$ is calculated using iterative procedure which involves the computation and inversion of Hessian matrix. The process is computationally intensive. Therefore, quasi-Newton approaches have been developed. They have good performance but poor accuracy due to approximations [12]. In comparison, RD has simple structure and can be suitable for online computation for UC problem.

\section{B. Unit Commitment model for Replicator Dynamics}

The objective of the UC model, as part of generation side management, is to minimize the total operational cost,

$$
C_{\text {total }}=\sum_{i=1}^{N} \sum_{k=1}^{T} F_{i}[k]+U_{i}[k]+D_{i}[k]
$$

over the scheduling horizon [1]. Where $F, U$ and $D$ stands for fuel, start-up and shut-down cost, respectively. The fuel cost is taken as a quadratic function of the power generated and is represented by the cost factor $c_{i}$ in (4).

The objective function in Eq. (9) is subject to the following constraints [13]: (i) System power balance, see Eq. (10); (ii)

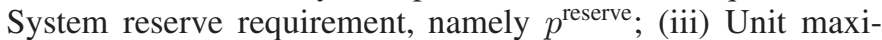
mum (resp. minimum) operating limits, denoted $p^{\text {nom }}$ (resp. $p^{\text {min }}$ ); (iv) Unit minimum-up (resp. down) time, $T_{\min }^{\text {up }}$ (resp. $T_{\text {min }}^{\text {down }}$ ); (v) Unit status restrictions (must run, unavailable, fixed production).

In order to facilitate the handling of constraints in the algorithm following (binary logic) states of each unit are defined: (i) $I_{i}[k]$ : Commitment state of the unit $i$ at $k^{\text {th }}$ hour; (ii) $I_{i}^{\text {start/stop }}[k]$ : Transition start-up (resp. shut-down); (iii) $X_{\mathrm{i}}^{\text {off }}[k]$ : time duration for which the unit $i$ has been switched off; These states are subsequently used in calculation of fitness function values.

The equality constraint, which is given as

$$
\sum_{i=1}^{N} p_{i}-\sum_{i=1}^{N} p_{i}^{\text {reserve }}=P_{d}+P_{L}=P_{d}^{\prime}
$$

is satisfied by the design of the average fitness function in Eq. (5) itself [8]. Here $P_{L}$ stands for the power loss and is computed using B-coefficient method [11]. The reserve power requirement from the unit is handled as reduction in its nominal rating:

$$
\left(p_{i}^{\text {nom }}-p_{i}^{\text {reserve }}\right) \rightarrow p_{i}^{\text {nom }}
$$

i.e. the reserve power can be made available (if required), by increasing the nominal rating of the unit within the available margin. The inequality constraints corresponding to the unit power limits are modeled as bounds on the fitness function:

$$
f_{i}^{\min } \leq f_{i} \leq f_{i}^{\max }
$$

It is to be noted that $f_{i}^{\max }$ corresponds to $p_{i}^{\min }$, see Eq. (4). The detection of a unit tending to shut-down or start-up is performed by implementing the following limit checks:

For shut-down:

$$
\begin{aligned}
\left(f_{i}>f_{i}^{\max }\right) & \wedge\left(\bar{f}>f_{i}^{\max }\right) \wedge\left(I_{i}[k-1]=1\right) \\
& \wedge\left(\sum_{\alpha=k-T_{\min }^{\mathrm{up}}}^{k-1} I_{i}[\alpha]=T_{\min }^{\mathrm{up}}\right) .
\end{aligned}
$$

For start-up:

$$
\begin{aligned}
\left(f_{i} \leq f_{i}^{\max }\right) & \wedge\left(\bar{f} \leq f_{i}^{\max }\right) \wedge\left(I_{i}[k-1]=0\right) \\
& \wedge\left(\sum_{\alpha=k-T_{\min }^{\text {down }}}^{k-1} I_{i}(\alpha)=T_{\text {min }}^{\text {down }}\right)
\end{aligned}
$$


where $\wedge$ symbolizes the logical \& operator. If the conditions in Eqs. (13) / (14) are met, then the shut-down/start-up costs are incorporated in the fitness function values as,

$$
f_{i}^{\prime}=f_{i}-I_{i}^{\text {start }}[k] D_{i}^{\text {marg }}+I_{i}^{\text {stop }}[k] U_{i}^{\text {marg }},
$$

where $D_{i}^{\mathrm{marg}}$ and $U_{i}^{\mathrm{marg}}$ stands for marginal shut-down cost and start-up cost, respectively. The start-up cost is modeled as

$$
U_{i}[k]=\left(1-e^{-X_{\mathrm{i}}^{\mathrm{off}}[k] / \tau}\right) U_{i}^{\mathrm{marg}},
$$

where $\tau$ is the unit start-up function time constant. The economic feasibility is then checked according to the respective shut-down and start-up inequality conditions,

$$
f_{i}^{\prime}>f_{i}^{\max }, f_{i}^{\prime} \leq f_{i}^{\max }
$$

The resulting algorithm is the bounded form of the original $\mathrm{RD}$ algorithm thus it retains the optimality characteristics.

\section{Stability of Equilibrium}

A strategy $i$ is said to be evolutionary stable if it is robust against the opposing strategies. According to the dynamicalsystems theory, a steady state is reached when the condition $\dot{x}_{i}=0$ is satisfied. In the context of RD, the right-hand side of Eq. (1) give us the stable vector field of the game, thus enabling to define the equilibrium.

For our model, the equilibrium is reached when each player plays its respective evolutionary stable strategy depicted by Eq. (6). The resultant equilibrium satisfy the criterion of asymptotic stability. Such that the solution path that starts close to equilibrium as result of minimal perturbation remain arbitrarily close and converges to the equilibrium in steady sate[11].

\section{RESULTS}

The proposed algorithm for UC has been applied on single area 26-bus power system [11] simulated in Matlab ${ }^{1}$ as test case for our studies. The details of the parameters can be found in Tab. I. The system is simulated for the demand data of one week duration that is hourly sampled. The spinning reserve requirement of $5 \%$ of total load has to be met for each time period.

The dynamics of the generation allocation to the six units in the test case is shown in Fig. 1, where the following observations can be made: (i) The shut-down (resp. start-up) of U4 and U6 occurs when they satisfy the minimum up-time constraint (resp. minimum down-time constraint) as described in Eq. (13) (resp. Eq. (14)); (ii) At time = 140 Hours, U4 is shutdown due to the low demand of energy. For ulterior times it is not turned on, despite the increase in demand. This behavior is due to the fact that the start-up cost makes U4 not a feasible candidate for start-up; (iii) For each dispatch the value committed to slack unit is verified by comparison with load flow result. In case of mismatch the dispatch process is repeated until the error is less than 0.001 .

${ }^{1}$ http: / / www . mathworks. com, MathWorks

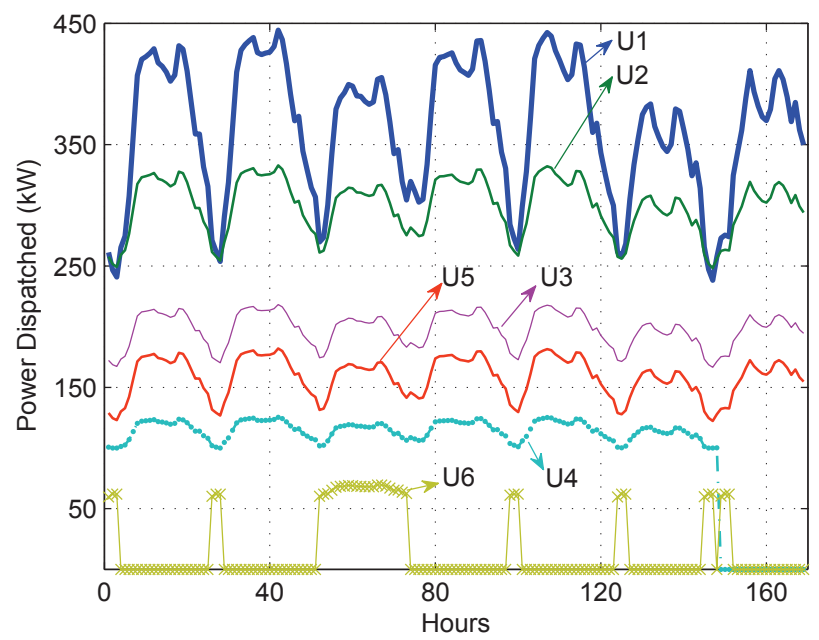

Fig. 1. Power dispatch dynamics for 26-bus power system using RD algorithm, where $\mathrm{U} 1$ is the slack unit

In Fig. 2-(a), It is observed that RD based optimal dispatch satisfy the equality constraints, Eq. (10). Both RD and NA are compared for the dispatch of U4, the accuracy of both algorithms is clearly demonstrated in Fig. 2-(b), the error is less than $1.12 \%$. The convergence accuracy of $1 e-005$ is used for both the algorithms during simulation.
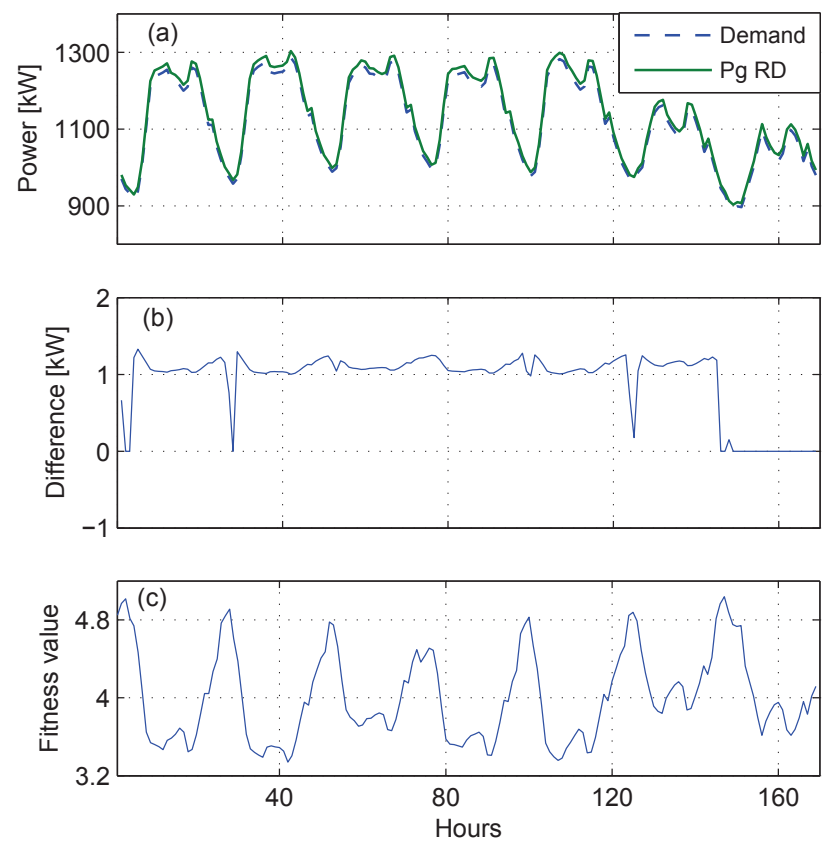

Fig. 2. (a) Comparison of the total power dispatched using RD Vs the total demand, Eq. (10). (b) Difference between power dispatched for the unit 4 between results of RD and NA algorithms. (c) Fitness function dynamics of the unit 2 for the whole demand profile

In Fig. 2-(c), the fitness function dynamics for a single unit $\mathrm{U} 2$ is shown for complete demand profile. It is observed that the fitness function remains within the bounds defined by operating limits of the unit. So the inequality constraint of Eq. (12) remains satisfied. Furthermore, the ramp limits of the 
TABLE I. SYSTEM DATA

\begin{tabular}{|c|c|c|c|c|c|c|c|c|}
\hline & $p^{\text {nom }}$ & $p^{\min }$ & $T_{\min }^{\mathrm{up}}$ & $T_{\min }^{\text {down }}$ & Cost factor $\left(c_{i}\right)$ & $D_{i}^{\operatorname{marg}}$ & $U_{i}^{\operatorname{marg}}$ & Start-up time constant $(\tau)$ \\
\hline Units & $k W$ & $k W$ & Hours & Hours & per-unit & per-unit & per-unit & Hours \\
\hline U1 & 850 & 100 & 6 & 5 & 0.143 & 0.14 & 0.2 & 4 \\
\hline $\mathrm{U} 2$ & 500 & 50 & 5 & 5 & 0.100 & 0.12 & 0.18 & 3 \\
\hline U3 & 300 & 50 & 5 & 4 & 0.117 & 0.10 & 0.16 & 3 \\
\hline U4 & 180 & 100 & 3 & 2 & 0.090 & 0.09 & 0.13 & 2 \\
\hline U5 & 320 & 50 & 4 & 4 & 0.095 & 0.11 & 0.15 & 3 \\
\hline U6 & 100 & 62 & 3 & 2 & 0.083 & 0.08 & 0.10 & 2 \\
\hline
\end{tabular}
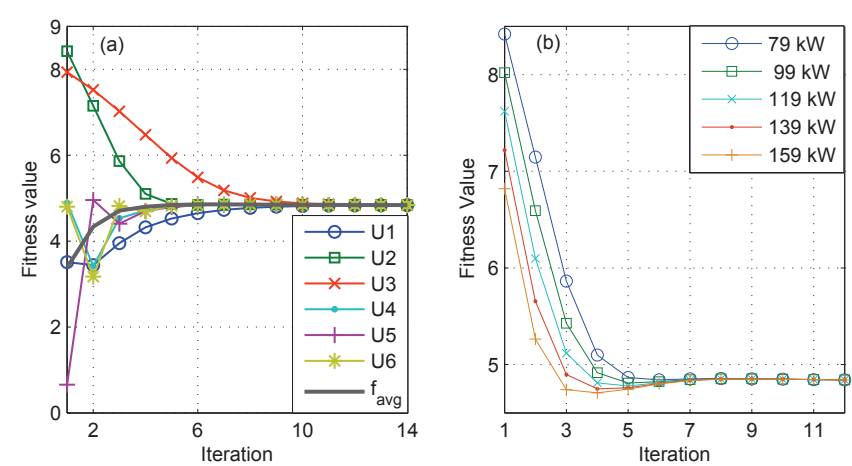

Fig. 3. For single instance [k]: (a) Evolution of fitness function of the units and average fitness value (where, $f_{\text {avg }}$ is the average fitness value). (b) The convergence of fitness function of $\mathrm{U} 2$ to average fitness function value for different initial conditions.

unit can be modeled as slope of the fitness function curve.

Fig. 3 discusses the dynamics of the fitness function computation. In Fig. 3-(a), the convergence of the fitness function values to the average fitness value is elaborated. This convergence happens in finite iterations and the convergence rate can be controlled by specifying the value of sampling time $T_{s}$ mentioned in Eq. (3). For the test case, it is fixed at 0.16 . Increasing the sampling time decreases the iteration count for convergence. But beyond a specific limit, the fitness value may start oscillating about the solution and do not satisfy the convergence accuracy criteria. In this work it is obtained experimentally. Fig. 3-(b) shows the robustness of $\mathrm{RD}$ approach for converging to the solution when started from different initial conditions. These results demonstrate the robustness of the algorithm, the only requirement is that the equality constraint should be satisfied at initial condition [8].

\section{CONCLUSION}

From the simulation results it is observed that the UC with the RD method shows good performance and meets the mentioned constraints. However, the ramping rate constraints and look ahead dispatch can further improve the results. The work done in this paper successfully shows the first level of the unit commitment optimization based on RD. It is observed that the convergence of fitness function is independent of initial value and happens in finite steps. The test case in this work assumes classical quadratic cost function structure. But the derivation is generic and can be used for other structures like piece-wise linear and polynomial functions. The structure of the algorithm has resemblance to secondary level control in Microgrid where the central node coordinates with local droop based controllers to deal with steady state deviations in frequency and voltage. Thus an additional level of dispatch based on this work can be a part of this secondary function.

Such an implementation at physical level requires sufficient bandwidth due to substantial data exchanged between nodes for each time instance $[k]$. Steps can be taken to improve the algorithm to reduce the communication bandwidth. The performance of the RD algorithm can be evaluated for more complex cases having, for example, coupled or discontinuous dynamic constraints. This work does not include the ramp rate constraints on the units. For future work, the unit specific coupled constraints and rate limit could be directly incorporated in the fitness functions. In this case, it may archly simplify the modeling of dynamic constraints. Furthermore, the convergence of this method can be improved by introducing a gain multiplier to Eq. (2).

\section{REFERENCES}

[1] N. Padhy, "Unit commitment-a bibliographical survey," Power Systems, IEEE Transactions on, vol. 19, no. 2, pp. 1196-1205, 2004.

[2] J. Hofbauer and K. Sigmund, "Evolutionary game dynamics," Bulletin of the American Mathematical Society, vol. 40, no. 04, pp. 479-520, Oct 2003.

[3] M. Carrion and J. Arroyo, "A computationally efficient mixed-integer linear formulation for the thermal unit commitment problem," Power Systems, IEEE Transactions on, vol. 21, no. 3, pp. 1371-1378, 2006.

[4] D. Zhai, A. Breipohl, F. Lee, and R. Adapa, "The effect of load uncertainty on unit commitment risk," Power Systems, IEEE Transactions on, vol. 9, no. 1, pp. 510-517, 1994.

[5] H.-T. Yang, P.-C. Yang, and C.-L. Huang, "Evolutionary programming based economic dispatch for units with non-smooth fuel cost functions," Power Systems, IEEE Transactions on, vol. 11, no. 1, pp. 112-118, 1996.

[6] H. Chen and X. Wang, "Cooperative coevolutionary algorithm for unit commitment," Power Systems, IEEE Transactions on, vol. 17, no. 1, pp. 128-133, 2002

[7] S. Frank, I. Steponavice, and S. Rebennack, "Optimal power flow: a bibliographic survey ii,” Energy Systems, vol. 3, no. 3, pp. 259-289, 2012.

[8] A. Pantoja and N. Quijano, "A population dynamics approach for the dispatch of distributed generators," Industrial Electronics, IEEE Transactions on, vol. 58, no. 10, pp. 4559-4567, 2011.

[9] K. Sigmund, The calculus of selfishness. Princeton University Press, 2010.

[10] D. Sun, B. Ashley, B. Brewer, A. Hughes, and W. F. Tinney, "Optimal power flow by newton approach," Power Apparatus and Systems, IEEE Transactions on, vol. PAS-103, no. 10, pp. 2864-2880, 1984

[11] H. Saadat, Power System Analysis. McGraw-Hill Higher Education, 2010.

[12] S. Frank, I. Steponavice, and S. Rebennack, "Optimal power flow: a bibliographic survey i," Energy Systems, vol. 3, no. 3, pp. 221-258, 2012.

[13] S. Kazarlis, A. Bakirtzis, and V. Petridis, "A genetic algorithm solution to the unit commitment problem," Power Systems, IEEE Transactions on, vol. 11, no. 1, pp. 83-92, 1996. 


\section{Workshop on Modeling and Simulation of Cyber-Physical Energy Systems (MSCPES)}

- IEEE

April 14, 2014, Berlin, Germany

Held as part of CPS Week, April 14-17, 2014, Berlin, Germany

Technically co-sponsored by the IEEE Industrial Electronics Society

ISBN: 978-1-4799-4702-7

IEEE Catalog Number: CFP1494U-ART

\section{PROCEEDINGS}

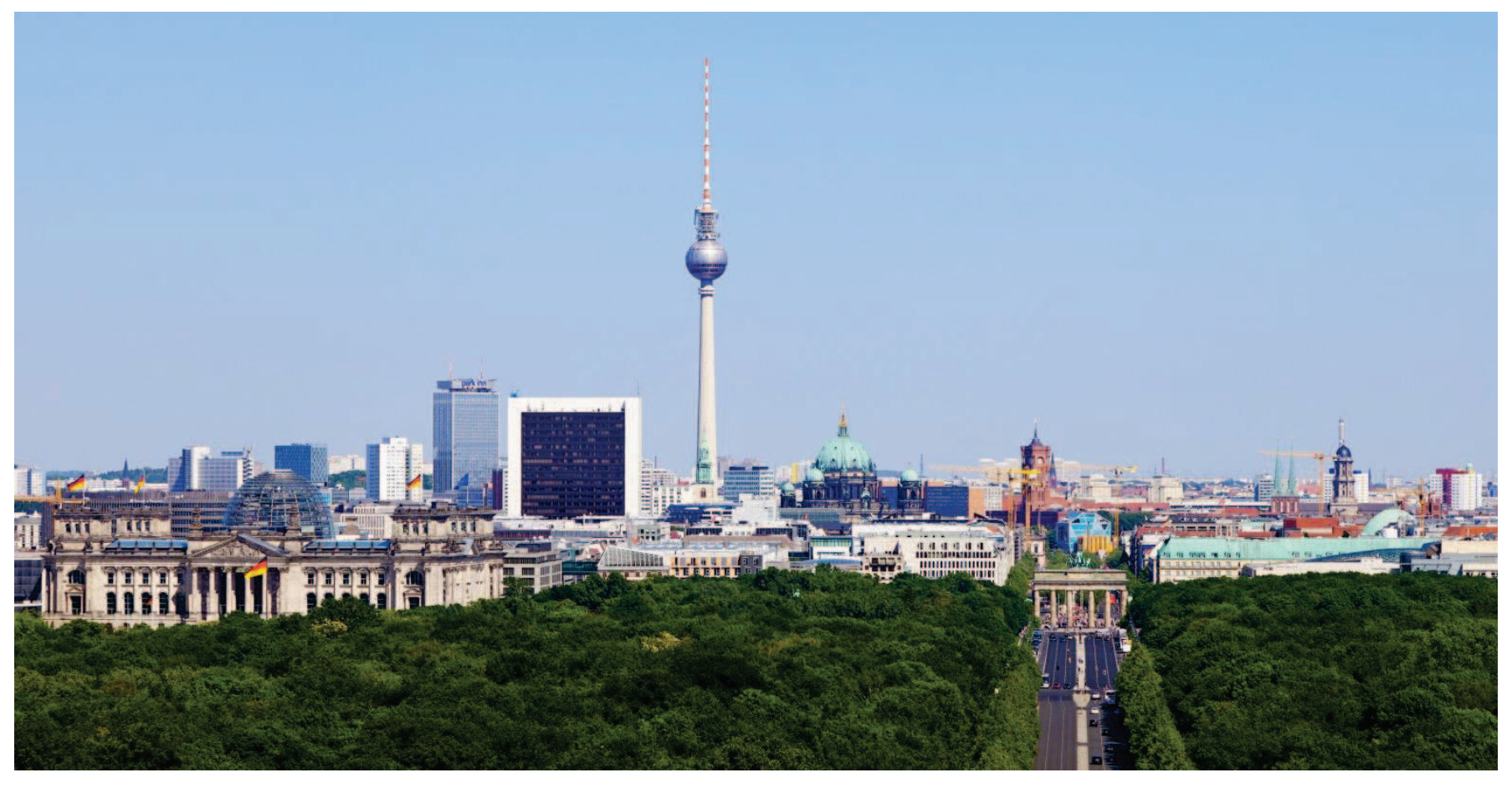

Photo (c) Thomas Wolf, www.foto-tw.de 